\title{
STEINER SYMMETRALS AND THEIR DISTANCE FROM A BALL
}

\author{
Gabriele BIANCHI AND PAOLO GRONCHI
}

\begin{abstract}
A BSTRACT. It is known that given any convex body $K \in \mathbb{R}^{n}$ there is a sequence of suitable iterated Steiner symmetrizations of $K$ that converges, in the Hausdorff metric, to a ball of the same volume. Hadwiger and, more recently, Bourgain, Lindenstrauss and Milman have given estimates from above of the number $N$ of symmetrizations necessary to transform $K$ into a body whose distance from the equivalent ball is less than an arbitrary positive constant.

In this paper we will exhibit some examples of convex bodies which are "hard to make spherical". For instance, for any choice of positive integers $n \geq 2$ and $m$, we construct an $n$-dimensional convex body with the property that any sequence of $m$ symmetrizations does not decrease its distance from the ball. A consequence of these constructions are some lower bounds on the number $N$.
\end{abstract}

\section{Introduction.}

Let $K$ be an $n$-dimensional convex body. It is known ([H1]) that there is a sequence of suitable iterated Steiner symmetrizations of $K$ that converges, in the Hausdorff metric, to a ball of the same volume.

In this paper we will study quantitative information on this convergence.

Let $\mathcal{K}_{1}$ denote the class of $n$-dimensional convex bodies whose volume equals $\kappa_{n}$, the volume of the unit ball $B_{n}$. For any $\varepsilon>0$ we denote by $N(n, \varepsilon)$ the minimum number of successive Steiner symmetrizations needed to transform any body in $\mathcal{K}_{1}$ into one whose Hausdorff distance from $B_{n}$ is $\leq \varepsilon$ (see the next section for definitions).

Hadwiger [H2] obtained an upper bound for $N(n, \varepsilon)$ : in the subclass of $\mathcal{K}_{1}$ of the bodies contained in a ball of radius $R$,

$$
N(n, \varepsilon) \leq(4 R \sqrt{n}+2 \varepsilon)^{n} \varepsilon^{-2 n}
$$

In the proof he uses some estimates of the number of small balls needed to cover a given cube; substituting them with better estimates available in the literature ([FTK]) one can improve the previous bound to $N(n, \varepsilon) \leq(4 R)^{n}(n \log n+n \log \log n+$ $4 n) \varepsilon^{-2 n}$.

More recently, Bourgain, Lindenstrauss and Milman [BLM] have shown that there exist universal constants $a$ and $c$ such that

$$
N(n, a) \leq c n \log n
$$

1991 Mathematics Subject Classification. 52A20, 46B07.

Key words and phrases. Steiner symmetrization, Hausdorff distance, Euclidean ball. 
Regarding $N(n, \varepsilon)$ for $\varepsilon$ small they prove that

$$
S_{v_{m}} \ldots S_{v_{1}}(K) \subset(1+\varepsilon) B_{n},
$$

with $m \leq c n \log n+f(\varepsilon) n$, for some function $f$ of $\varepsilon\left(S_{v_{i}}\right.$ denote suitable Steiner symmetrizations), where $n$ is larger than a given function of $\varepsilon$ that grows unboundedly as $\varepsilon \rightarrow 0$. The asymptotic behaviour of $f(\varepsilon)$ as $\varepsilon \rightarrow 0$ is $f(\varepsilon) \approx e^{|\log \varepsilon| / \varepsilon^{2}}$. Their paper does not contain results regarding the other inclusion, that of $(1-\varepsilon) B_{n}$ in $S_{v_{m}} \ldots S_{v_{1}}(K)$.

Finally, we mention that Tsolomitis $[\mathrm{T}]$ has studied similar problems for some generalizations of the Steiner symmetrization.

In this paper we seek lower bounds on the function $N(n, \varepsilon)$. These bounds will be consequences of the existence of some convex bodies which are "hard to make spherical".

Theorem 1. Let $n \geq 2$. Given any positive integer $m$, there exists a non-spherical origin-symmetric convex body $H$ in $\mathbb{R}^{n}$ such that for any choice of $m$ directions $v_{1}$, $v_{2}, \ldots, v_{m}$ the body $S_{v_{m}} \ldots S_{v_{1}}(H)$ has the same inner and outer radius as $H$.

We recall that the inner radius $r$ and outer radius $R$ of a convex body $H$ are respectively the largest radius of a ball contained in $H$ and the smallest radius of a ball containing $H$. When the body is origin symmetric these balls are centered at the origin and the Hausdorff distance from $H$ to $B_{n}$ is $\max \{1-r, R-1\}$.

An immediate consequence of Theorem 1 is that it is not possible to reduce the Hausdorff distance of $H$ from the ball of the same volume using at most $m$ symmetrizations.

Corollary 2. Let $n \geq 2$. Given any positive integer $m$ there exists a non-spherical origin-symmetric body $H$ in $\mathbb{R}^{n}$ of volume $\kappa_{n}$ such that for any choice of $m$ directions $v_{1}, v_{2}, \ldots, v_{m}$ the body $S_{v_{m}} \ldots S_{v_{1}}(H)$ has the same Hausdorff distance from $B_{n}$ as $H$.

Estimates of the distance from $B_{n}$ of these, or similar, bodies give estimates of $N(n, \varepsilon)$ from below.

Theorem 3. Let $N(n, \varepsilon)$ be the function defined in (2.1) and let $n \geq 2$. Then

$$
N(n, \varepsilon) \geq \frac{\log (\log (1 / \varepsilon))}{\log 2}(1+o(1)) \quad \text { as } \varepsilon \rightarrow 0 .
$$

When $n$ is large compared to $m$, bodies like those in Corollary 2 can be easily constructed. Let $n \geq m+2$ and let $B_{n-1}$ be the intersection of the unit ball $B_{n}$ with a hyperplane through the origin. Let $R>2$ be arbitrary and let $H$ be the convex hull of $R B_{n-1}$ and $r B_{n}$, with $0<r<R$ such that $\operatorname{vol}(H)=\kappa_{n}$. The Hausdorff distance of $H$ from $B_{n}$ is not reduced by any $m$ symmetrizations (since symmetrizations with respect to directions $v_{1}, \ldots, v_{m}$ leave the points in $R B_{n-1} \cap v_{1}^{\perp} \cap \cdots \cap v_{m}^{\perp}$ unchanged, where $\left.v^{\perp}=\left\{x \in \mathbb{R}^{n}:\langle x, v\rangle=0\right\}\right)$. This distance is $R-1$ and so it can be made arbitrarily large. Therefore

$$
N(n, \varepsilon) \geq n-1 \quad \forall \varepsilon>0 .
$$

Finally, we would like to mention a technical result which might be of interest in itself. Proposition 4, in a particular case, implies the following statement. Given $v \in S^{n-1}$ we denote by $\pi_{v}$ the reflection with respect to $v^{\perp}$. 
Proposition 4 (in the case of two directions). Let $n \geq 2$. There exist arbitrarily small subsets $A$ of $S^{n-1}$ such that for any choice of the directions $v_{1}$, $v_{2} \in S^{n-1}$,

$$
\left(A \cap \pi_{v_{1}}(A)\right) \cap \pi_{v_{2}}\left(A \cap \pi_{v_{1}}(A)\right) \neq \emptyset
$$

This result is related to various problems, for instance to additive properties of sequences of integers studied by P. Erdös (see [HR], Ch. 3).

\section{Preliminaries.}

Given a direction $v \in S^{n-1}=\left\{x \in \mathbb{R}^{n}:\|x\|=1\right\}$, Steiner symmetrization along $v$ is the mapping that associates to each convex body $K \subset \mathbb{R}^{n}$ the unique convex body $S(K)$ with the following two properties:

(1) given any line $l$ parallel to $v, K \cap l$ and $S(K) \cap l$ are either both empty or are segments of the same length;

(2) given any line $l$ parallel to $v, S(K) \cap l$ is symmetric with respect to $v^{\perp}$.

This mapping is denoted by $S_{v}$ and the image $S_{v}(K)$ of $K$ is called the Steiner symmetral of $K$ along $v$.

Let $d_{H}(\cdot, \cdot)$ denote the Hausdorff distance. We define

$$
N(n, \varepsilon)=\sup _{K \in \mathcal{K}_{1}} \inf \left\{m \in \mathbb{N}: \exists v_{1}, \ldots, v_{m} \in S^{n-1} \text { with } d_{H}\left(B_{n}, S_{v_{m}} \ldots S_{v_{1}}(K)\right) \leq \varepsilon\right\}
$$

We now describe the ideas behind the construction of the body $H$ in Theorem 1 in the simple case of an origin-symmetric convex body $K$ such that any single symmetrization does not decrease its outer radius.

For any $z \in \mathbb{R}^{n}$ and for any direction $v \in S^{n-1}$, let $\pi_{v}(z)=z-2\langle z, v\rangle v$. We call $\pi_{v}(z)$ the point corresponding to $z$ along the direction $v$. Note that $\pi_{v}(z)$ and $z$ have the same distance from the origin. Moreover, if $R$ denotes the outer radius of $K$,

$$
z \in S_{v}(K) \cap R S^{n-1} \quad \text { if and only if } \quad z, \pi_{v}(z) \in K \cap R S^{n-1} .
$$

Clearly, the compact set $K$ has the property that no symmetrization decreases its outer radius if and only if $S_{v}(K) \cap R S^{n-1} \neq \emptyset$ for any direction $v$; this fact, due to (2.2), holds if and only if for any direction $v$ there exists $z \in K \cap R S^{n-1}$ such that $\pi_{v}(z) \in K \cap R S^{n-1}$. Thus the required property can be rephrased in terms which involve only the intersection of $K$ with the sphere with equal outer radius. Let $A$ be an origin-symmetric subset of $S^{n-1}$ such that

$$
\text { for any direction } v \text { there exists } z \in A \text { such that } \pi_{v}(z) \in A \text {. }
$$

Then its convex hull is such that no symmetrization decreases its outer radius. To construct a convex body whose outer and inner radius remain unchanged it suffices to construct two closed disjoint subsets $A$ and $B$ of $S^{n-1}$ that satisfy (2.3) and to consider the convex hull of $r S^{n-1}$ and $R B$ for some $0<r<R$, with $R / r$ so close to 1 that the boundary of this convex set contains $r A$.

If its $(n-1)$-dimensional Hausdorff measure is large enough, any subset of $S^{n-1}$ satisfies (2.3), but we are interested mainly in subsets whose measure is small. Fig. 1 shows some subsets of $S^{1}$ that satisfy (2.3). 
Fig.1

The set $A$ is $[0, \pi / 2] \cup[\pi, 3 \pi / 2]$; the set $B$ is obtained by removing the middle third of each interval of $A$, as in a Cantor set construction. Note that one can iterate this procedure and obtain sets of arbitrarily small measure that still satisfy (2.3). Furthermore this property also passes to the limit, providing us with an example of set of zero measure which determines all possible directions. Using polar coordinates one can easily check the latter statement. Everything can be 
deduced from the following well-known property of the Cantor set $E$. Every point in $[0,1]$ is the midpoint of a segment with endpoints in $E$, that is $E+E=[0,2]$. The set $C$ in Fig. 1 is defined by $C=\{\pi / 4,5 / 4 \pi\} \cup[7 / 12 \pi, 11 / 12 \pi] \cup[19 / 12 \pi, 23 / 12 \pi]$. Note that $B$ and $C$ are disjoint.

Given an ordered set $V$ of $m$ directions $v_{1}, v_{2}, \ldots, v_{m}$ in $S^{n-1}$, for any $x \in \mathbb{R}^{n}$ we call the orbit of $x$ the set $O_{V}(x)$ defined by

$$
O_{V}(x)=\left\{x, \pi_{v_{j_{s}}}\left(\pi_{v_{j_{s-1}}}\left(\ldots \pi_{v_{j_{1}}}(x)\right)\right), \text { for any } 1 \leq j_{1}<j_{2}<\cdots<j_{s} \leq m\right\} \text {. }
$$

All these points have the same distance from the origin. It is straightforward to check that if $r$ and $R$ are respectively the inner and outer radius of the originsymmetric convex body $K$ then

$$
z \in S_{m} \ldots S_{1}(K) \cap R S^{n-1} \quad \text { if and only if } \quad O_{V}(z) \subset K \cap R S^{n-1}
$$

and

$$
z \in \partial\left(S_{m} \ldots S_{1}(K)\right) \cap r S^{n-1} \quad \text { if and only if } \quad O_{V}(z) \subset \partial K \cap r S^{n-1}
$$

To construct the set $H$ of Theorem 1 we will construct subsets $A$ of $S^{n-1}$ with the property that

for any choice of the sequence $V$ of $m$ directions $v_{1}, v_{2}, \ldots, v_{m}$ there exists $x \in A$ such that $O_{V}(x) \subset A$.

We call this property the $m$-orbit property. Condition (2.3) expresses the 1-orbit property.

Proposition 4. For any positive integers $n \geq 2$ and $m$ there exist subsets of $S^{n-1}$ of arbitrarily small $\mathcal{H}^{n-1}$-measure which satisfy the m-orbit property.

Here $\mathcal{H}_{n-1}$ stands for $(n-1)$-dimensional Hausdorff measure.

\section{A discretization of the problem.}

In order to construct the sets $K \cap r S^{n-1}$ and $K \cap R S^{n-1}$, we solve a similar problem in the discrete case. We use an argument introduced by Erdös in the study of additive properties of sequences (see $[\mathrm{HR}], \mathrm{Ch} .3$ ). In the planar case, expressing the set $A$ in terms of polar coordinates, one has the following discretized version of the 1-orbit property: given a positive integer $M, A+A=\{0, \ldots, M-1\}$ mod $M$. Erdös studied the problem of finding "small" increasing subsequences $A$ of $\mathbb{N}$ such that $A+A=\mathbb{N}$ and gave a probabilistic proof of the existence of such subsequences.

If $x, y \in S^{n-1}$ we denote by $d(x, y)$ their geodesic distance. As usual, we write $d(x, A)$ for $\inf _{a \in A} d(x, a)$. Given $\delta>0$, consider a finite subset $T$ of $S^{n-1}$ with the following properties:

$$
\inf _{x \in T} d(x, T \backslash\{x\}) \geq 2 \delta, \quad \sup _{y \in S^{n-1}} d(y, T)<2 \delta .
$$

$T$ is called a $\delta$-net and it will play the role of a discretization of the sphere. For instance, the set of the centers of a densest packing of $S^{n-1}$ with spherical caps having radius $\delta$ is a $\delta$-net. 
Let $t=|T|$; about the relation between $t$ and $\delta$ one can observe the following. (3.1) implies that the union of the spherical caps centered in $T$ and with radius $\delta$ constitute a packing of $S^{n-1}$, while the union of those with radius $2 \delta$ constitute a covering. Let $\sigma_{n-1}(\phi)$ denote the $(n-1)$-dimensional Hausdorff measure of the spherical cap in $S^{n-1}$ with radius $\phi$. Since

$$
\sigma_{n-1}(\phi)=(\sin \phi)^{n-1}(n-1) \kappa_{n-1} \int_{0}^{1} \frac{t^{n-2}}{\sqrt{1-t^{2} \sin ^{2} \phi}} d t
$$

we have

$$
\begin{aligned}
& t \leq \bar{t}(\delta):=c(n) \sin ^{1-n} \delta \quad \text { and } \\
& t \geq \underline{t}(\delta):=c(n) \sin ^{1-n} 2 \delta \cos 2 \delta
\end{aligned}
$$

where $c(n)=n \kappa_{n} / \kappa_{n-1}$. The relation between $t$ and $\delta$ in the case of the densest packing has been studied thoroughly; see [FTK, p. 824] for better bounds on $t$.

For each $v \in T$ we define a map $\pi_{v}{ }^{\prime}: T \rightarrow T$, the map which corresponds in this setting to the reflection with respect to $v^{\perp}$, as follows. To every $z \in T$ we associate a point $w$ of $T$ that minimizes the distance of $\pi_{v}(z)$ from $T$, with $d\left(z_{v}, w\right)=d\left(z_{v}, T\right)$. From (3.1), we have $d\left(\pi_{v}{ }^{\prime}(z), \pi_{v}(z)\right)<2 \delta$.

The map $\pi_{v}{ }^{\prime}$ is not necessarily injective, but an upper bound for the cardinality of $\pi_{v}^{\prime-1}(x)$ can be easily computed. This follows from the observation that if $z_{1}$, $z_{2}, \ldots, z_{b}$ have the same image $w$ then the $b$ spherical caps centered at the points $\pi_{v}\left(z_{i}\right)$ and with radius $\delta$ are disjoint and all contained in the spherical cap centered at $w$ and of radius $3 \delta$. The measure of these balls implies that $b \sigma_{n-1}(\delta) \leq \sigma_{n-1}(3 \delta)$ and thus

$$
b<\left(\frac{\sin 3 \delta}{\sin \delta}\right)^{n-1} \frac{1}{\cos 3 \delta}
$$

Fix $m \in \mathbb{N}$. For every sequence $W=w_{1}, w_{2}, \ldots, w_{m}$ of $m$ directions in $T$ we define the discretized $W$-orbit of a point $x \in T$ as follows:

$$
O_{W}^{\prime}(x)=\left\{x, \pi_{w_{j_{s}}}{ }^{\prime}\left(\pi_{w_{j_{s-1}}}{ }^{\prime}\left(\ldots \pi_{w_{j_{1}}}{ }^{\prime}(x)\right)\right) \text {, with } 1 \leq j_{1}<j_{2}<\cdots<j_{s} \leq m\right\} .
$$

Thus $O_{W}^{\prime}(x)$ is the subset of $T$ consisting of $x, \pi_{w_{1}}{ }^{\prime}(x), \pi_{w_{2}}{ }^{\prime}(x), \pi_{w_{2}}{ }^{\prime}\left(\pi_{w_{1}}{ }^{\prime}(x)\right)$, and so on. Notice that $\left|O_{W}^{\prime}(x)\right| \leq 2^{m}$.

Lemma 5. Suppose that for any choice of $v, z \in T$ the set $\pi_{v}^{\prime-1}(z)$ contains at most $b(n)$ elements. Then, for every $W \in T^{m}$, there exist at least $\left[t /(2 b(n)+2)^{m}\right]$ disjoint discretized $W$-orbits.

Proof. For every $x \in T$, consider the sets

$$
D(x)=\left\{y \in T: x \in O_{W}^{\prime}(y)\right\}
$$

and

$$
E(x)=\bigcup_{\substack{z \in O_{W}^{\prime}(x) \\ 6}} D(z)
$$


Let us estimate the cardinality of $E(x)$. An element $y$ belongs to $E(x)$ if and only if there are indices $1 \leq i_{1}<i_{2}<\cdots<i_{q} \leq m, 1 \leq j_{1}<j_{2}<\cdots<j_{s} \leq m$ such that

$$
y \in \pi_{w_{i_{1}}}^{\prime-1}\left(\pi_{w_{i_{2}}}^{\prime-1}\left(\ldots \pi_{w_{i_{q}}}^{\prime-1}\left(\pi_{w_{j_{s}}}{ }^{\prime}\left(\pi_{w_{j_{s-1}}}{ }^{\prime}\left(\ldots \pi_{w_{j_{1}}}{ }^{\prime}(x)\right)\right)\right)\right)\right) .
$$

Thus $|E(x)| \leq(2 b(n)+2)^{m}$. Choose any $x_{1}$ and any $x_{2} \notin E\left(x_{1}\right)$; by definition $O_{W}^{\prime}\left(x_{1}\right) \cap O_{W}^{\prime}\left(x_{2}\right)=\emptyset$. Choosing $x_{3} \notin E\left(x_{1}\right) \cup E\left(x_{2}\right)$ we have that $O_{W}^{\prime}\left(x_{1}\right) \cap$ $O_{W}^{\prime}\left(x_{2}\right) \cap O_{W}^{\prime}\left(x_{3}\right)=\emptyset$. One can repeat this argument choosing $x_{1}, x_{2}, \ldots, x_{q}$ until the set $\bigcup_{i=1}^{q} E\left(x_{i}\right)$ coincides with $T$. Since the cardinality of this union is at most $q(2 b(n)+2)^{m}$ this may happen only when $q>t /(2 b(n)+2)^{m}$. This concludes the proof.

Now our aim is to construct a small subset of $T$ such that, for every $W \in T^{m}$, it contains a $W$-orbit. We shall use a probabilistic argument.

Let $p_{1}, p_{2}, \ldots, p_{t}$ denote the points of $T$.

Fix $\alpha \in(0,1)$ and let $\Gamma$ be the set of the subsets of $T$. We define a probability measure $\mathcal{P}$ on $\Gamma$ by $\mathcal{P}(A)=\alpha^{|A|}(1-\alpha)^{t-|A|}$ for $A \in \Gamma$.

If we identify the set $A$ with the element $\left(a_{1}, a_{2}, \ldots, a_{t}\right) \in \Omega=\{0,1\}^{t}$ such that $a_{i}=1$ if $p_{i} \in A$ and $a_{i}=0$ otherwise, then $(\Omega, \mathcal{G}, \mathcal{P})$, where $\mathcal{G}$ is the set of subsets of $\Omega$, is the Bernoulli probability with parameter $\alpha$. Let us now evaluate the probability that a random subset $A$ of $T$ contains a fixed $B \in \Gamma$. We can write

$$
\begin{aligned}
\mathcal{P}(A \in \Gamma: A \supset B) & =\sum_{A \supset B} \mathcal{P}(A)=\sum_{k=|B|}^{t} \sum_{A \supset B,|A|=k} \alpha^{k}(1-\alpha)^{t-k} \\
& =\sum_{k=|B|}^{t}\left(\begin{array}{c}
t-|B| \\
k-|B|
\end{array}\right) \alpha^{k}(1-\alpha)^{t-k} \\
& =\alpha^{|B|} \sum_{k=0}^{t-|B|}\left(\begin{array}{c}
t-|B| \\
k
\end{array}\right) \alpha^{k}(1-\alpha)^{t-|B|-k}=\alpha^{|B|} .
\end{aligned}
$$

As a consequence, if $B_{1}, B_{2}, \ldots, B_{k}$ are disjoint subsets of $T$, then all the events $\left\{A \in \Gamma: A \supset B_{k}\right\}$ are independent. Indeed, for every $i_{1}, i_{2}, \ldots, i_{s} \in\{1,2, \ldots, k\}$, we have

$$
\begin{gathered}
\mathcal{P}\left(\left\{A: A \supset B_{i_{1}}\right\} \cap \cdots \cap\left\{A: A \supset B_{i_{s}}\right\}\right)=\mathcal{P}\left(\left\{A: A \supset \bigcup_{r=1}^{s} B_{i_{r}}\right\}\right) \\
=\alpha^{\left|\bigcup_{r=1}^{s} B_{i_{r}}\right|}=\alpha^{\sum_{r=1}^{s}\left|B_{i_{r}}\right|}=\prod_{r=1}^{s} \mathcal{P}\left(\left\{A: A \supset B_{i_{r}}\right\}\right) .
\end{gathered}
$$

Thus, given $W \in T^{m}$ and $x \in T$, the probability that $A$ contains the $W$-orbit of $x$ is

$$
\mathcal{P}\left(A \in \Gamma: A \supset O_{W}^{\prime}(x)\right)=\alpha^{\left|O_{W}^{\prime}(x)\right|} \geq \alpha^{2^{m}}
$$

By Lemma 5, there exist $x_{1}, x_{2}, \ldots, x_{k}$ such that $O_{W}^{\prime}\left(x_{i}\right) \cap O_{W}^{\prime}\left(x_{j}\right)=\emptyset$, for $i \neq j$, 
and $k \geq\left[t /(2 b(n)+2)^{m}\right]$. Hence, for a fixed $W$, we can write

$$
\begin{aligned}
\mathcal{P}\left(\cup_{x \in T}\left\{A: A \supset O_{W}^{\prime}(x)\right\}\right) & \geq \mathcal{P}\left(\cup_{i=1}^{k}\left\{A: A \supset O_{W}^{\prime}\left(x_{i}\right)\right\}\right) \\
& =1-\mathcal{P}\left(\cap_{i=1}^{k}\left\{A: A \nsupseteq O_{W}^{\prime}\left(x_{i}\right)\right\}\right) \\
& =1-\prod_{i=1}^{k} \mathcal{P}\left(\left\{A: A \nsupseteq O_{W}^{\prime}\left(x_{i}\right)\right\}\right) \\
& \geq 1-\prod_{i=1}^{k}\left(1-\alpha^{2^{m}}\right) \\
& \geq 1-\left(1-\alpha^{2^{m}}\right)^{\left[t /(2 b(n)+2)^{m}\right]},
\end{aligned}
$$

where we have used the fact that all the events $\left\{A \in \Gamma: A \supset O_{W}^{\prime}\left(x_{i}\right)\right\}$ are independent since the orbits $O_{W}^{\prime}\left(x_{i}\right)$ are disjoint, and so are their complementary sets.

Lemma 6. Let $T$ be a $\delta$-net and let $t=|T|$. Let $f$ be an integer in $[1, t]$. The $\mathcal{P}$-probability that a subset $A$ of $T$ has $|A|<f$ and contains a $W$-orbit for every $W \in T^{m}$ is greater than or equal to

$$
1-t^{m}\left(1-\alpha^{2^{m}}\right)^{\left[t /(2 b(n)+2)^{m}\right]}-\sum_{i=f}^{t}\left(\begin{array}{l}
t \\
i
\end{array}\right) \alpha^{i}(1-\alpha)^{t-i}
$$

If this number is positive then there exists such a set $A$.

Proof. Passing to the complementary sets let us estimate

$$
\mathcal{P}\left(\bigcup_{W \in T^{m}}\left\{A: A \nsupseteq O_{W}^{\prime}(x) \forall x \in T\right\} \cup\{A:|A| \geq f\}\right) .
$$

The inequality (3.5) implies that for each $W \in T^{m}$,

$$
\mathcal{P}\left(\left\{A: A \nsupseteq O_{W}^{\prime}(x) \forall x \in T\right\}\right) \leq\left(1-\alpha^{2^{m}}\right)^{\left[t /(2 b(n)+2)^{m}\right]} .
$$

Thus the quantity in (3.7) is less than or equal to

$$
\mathcal{P}(\{A:|A| \geq f\})+\sum_{W \in T^{m}}\left(1-\alpha^{2^{m}}\right)^{\left[t /(2 b(n)+2)^{m}\right]} .
$$

The fact that $T^{m}$ contains $t^{m}$ elements and standard formulas for the binomial distribution conclude the proof.

Let us choose in (3.6) $f=\alpha t$, the expected value of the cardinality of a set in $T$. If $\alpha$ and $m$ are fixed and $\delta \rightarrow 0$ then the expression in (3.6) becomes positive. Indeed the second term tends to 0 while the third one tends to $1 / 2$, as the approximation of the binomial distribution with a normal one shows. The expression in (3.6) also becomes positive as $\delta \rightarrow 0$ if we choose $\alpha=t^{-\beta}$ for $0<\beta<1 / 2^{m}$. Indeed the second term again converges to 0 and the third to $1 / 2$. Note that the approximation of the binomial with a normal distribution is still valid since $\alpha(1-\alpha) t \rightarrow \infty$. 


\section{Existence results.}

Given a subset $A \subset S^{n-1}$ and a positive constant $a$ we denote by $(A)_{a}=\{x \in$ $\left.S^{n-1}: d(x, A)<a\right\}$ the open $a$-neighborhood of $A$.

Lemma 7. Let $T$ be a $\delta$-net for some $\delta>0$, and let $A$ be a subset of $T$ that contains a discretized $W$-orbit for any $W \in T^{m}$. Then the subset $(A)_{6 m \delta}$ of $S^{n-1}$ satisfies the m-orbit property.

Proof. Let $V=v_{1}, v_{2}, \ldots, v_{m}$ be any sequence of $m$ directions in $S^{n-1}$, for each $i$ let $w_{i} \in T$ be such that $d\left(v_{i}, w_{i}\right)<2 \delta$ and let $W=\left\{w_{1}, w_{2}, \ldots, w_{m}\right\}$. Then for any $y \in T$ we have $d\left(\pi_{v_{i}}(y), \pi_{w_{i}}{ }^{\prime}(y)\right)<6 \delta$. There exists $x \in A$ such that $O_{W}^{\prime}(x) \subset A$; it is straightforward to check that $O_{V}(x) \subset(A)_{6 m \delta}$.

Lemma 8. Let $A \subset S^{n-1}$ be such that $\mathcal{H}_{n-1}\left(S^{n-1} \backslash A\right)<n \kappa_{n} / 2^{m}$. Then $A$ satisfies the $m$-orbit property.

Proof. Let $B=S^{n-1} \backslash A$ and let $\overleftarrow{V}=v_{m}, v_{m-1}, \ldots, v_{1}$ be $V$ in the opposite order. The set $O_{\overleftarrow{V}}(B)=\bigcup_{y \in B} O_{\overleftarrow{V}}(y)$ is the union of at most $2^{m}$ sets obtained from $B$ through a finite number of reflections and thus of sets which have the same measure as $B$. This implies that $\mathcal{H}_{n-1}\left(O_{\overleftarrow{V}}(B)\right)<n \kappa_{n}$ and thus that $S^{n-1} \backslash O_{\overleftarrow{V}}(B) \neq \emptyset$. If $x \in S^{n-1} \backslash O_{\overleftarrow{V}}(B)$ then $O_{V}(x) \subset S^{n-1} \backslash B=A$.

Proof of Proposition 4. Let $T \subset S^{n-1}$ be a $\delta$-net for a given $\delta>0$ and let $t=|T|$.

Let $\alpha=t^{-\beta}$, for some $0<\beta<1 / 2^{m}$ and $f=\alpha t$. As observed after the proof of Lemma 6 , if $t$ is large enough then the probability in (3.6) is positive. Therefore, by Lemma 6 , there exists a subset $A^{\prime}$ of $T$ which contains a $W$-orbit for each $W \subset T^{m}$. Let $A=\left(A^{\prime}\right)_{6 m \delta}$; by Lemma 7 it satisfies the $m$-orbit property.

Let us estimate $\mathcal{H}_{n-1}(A)$. We have that $\mathcal{H}_{n-1}(A) \leq\left|A^{\prime}\right| \sigma_{n-1}(6 m \delta) \leq t^{1-\beta} \sigma_{n-1}(6 m \delta)$ which, due to (3.2) and (3.3), becomes arbitrarily small as $\delta \rightarrow 0$.

Proof of Theorem 1. Let $A$ be a subset of $S^{n-1}$ with the $m$-orbit property and $\mathcal{H}_{n-1}(A) \leq n \kappa_{n} / 2^{m+1}$. Without loss of generality we may assume that $A$ is origin symmetric. By continuity it is possible to choose a positive number $a$ such that

$$
\mathcal{H}_{n-1}\left((A)_{a}\right) \leq \frac{n \kappa_{n}}{2^{m}}
$$

We claim that if the two positive numbers $r, R$ are such that $r / R<\cos a$ then, defining $H$ as the convex hull of $r S^{n-1}$ and of $R A^{\prime}$, we have that $H$ is origin symmetric, that its inner and outer radius are respectively $r$ and $R$, that $H \cap$ $R S^{n-1}=R A^{\prime}$ and $\partial H \supset r B$. The only assertion that needs to be proved is that $\partial H \supset r B$. Let $x \in r B$ and let $\pi$ be a hyperplane supporting $r S^{n-1}$ at $x$. The intersection of this hyperplane with $R S^{n-1}$ is a spherical cap with radius $\arccos (r / R)$. Therefore, for our choice of $r$ and $R$, the spherical cap cut by $\pi$ on $R S^{n-1}$ does not contain points of $A^{\prime}$. This implies that $\pi$ supports $R A^{\prime}$ and thus also $H$.

As observed in Section 2 the set $H$ satisfies the claim of Theorem 1 if and only if both the intersections of $\partial H$ with the boundary of the outer and inner spheres satisfy the $m$-orbit property. This is true for the outer sphere by construction, while for the inner sphere it follows from Lemma 8 and (4.1).

Remark. As it is clear from the previous proof, $a$ is a "measure" of the distance of $H$ from the equivalent sphere. This constant depends on $n$ and $m$. In the next section we try to maximize this distance for bodies similar to $H$. 


\section{Lower bounds.}

If $\varepsilon(n, m)$ denotes the Hausdorff distance from $B_{n}$ of the body $H \in \mathcal{K}_{1}$ constructed in the proof of Theorem 1 , then $N(n, \varepsilon(n, m))>m$. Thus, estimates of $\varepsilon(n, m)$ provide a lower bound for $N(n, \varepsilon)$. However we can get some better estimates if we relax the hypothesis that both the outer and inner radii remain unchanged. We shall construct convex bodies in $\mathcal{K}_{1}$ so that $m$ arbitrary Steiner symmetrizations do not reduce their outer radii and furthermore their Hausdorff distance from $B_{n}$ is attained by the outer ball. In order to do this we need the following lemma.

Lemma 9. Suppose that $r B_{n} \subset K \subset R B_{n}$. If $\mathcal{H}_{n-1}\left(K \cap s S^{n-1}\right) \leq n \kappa_{n} s^{n-1} / 2$ for every $s \in(r, R]$ and $\operatorname{vol}\left(c B_{n}\right)=\operatorname{vol}(K)$, then $c \leq(r+R) / 2$.

Proof. Consider the radial function $\rho_{K}$ of $K$, defined by

$$
\rho_{K}(u)=\max \{\lambda \in \mathbb{R}: \lambda u \in K\} .
$$

Taking into account the convexity of $K$ and the assumption $r B_{n} \subset K$, it is easy to see that $\rho_{K}$ is a Lipschitz function.

Now fix $w \in S^{n-1}$ and consider the function $\tau: S^{n-1} \rightarrow \mathbb{R}$ defined by

$$
\tau(z)= \begin{cases}r & \text { if }\langle z, w\rangle \leq 0 \\ \min \left\{R, \sqrt{r^{2}+\langle z, w\rangle^{2}}\right\} & \text { if }\langle z, w\rangle \geq 0 .\end{cases}
$$

Notice that $\tau$ is the radial function of a convex body $C$ of revolution, that can be decomposed into half a ball of radius $r$, a cylinder and a cap of radius $R$.

Let

$$
\begin{gathered}
M_{s}=\left\{z \in S^{n-1}: \rho_{K}(z)>s\right\}, \forall s \in[0, R], \\
L_{s}=\left\{z \in S^{n-1}: \tau(z)>s\right\}, \forall s \in[0, R] \\
m(s)=\mathcal{H}_{n-1}\left(M_{s}\right)
\end{gathered}
$$

and

$$
l(s)=\mathcal{H}_{n-1}\left(L_{s}\right)
$$

We want to prove that

$$
m(s) \leq l(s), \forall 0 \leq s \leq R .
$$

By our assumptions, (5.1) is true for $0 \leq s \leq r$. For larger values of $s,(5.1)$ can be proved by means of the isoperimetric inequality.

Assume $\rho_{K}\left(z^{\prime}\right)=s^{\prime}>r$. By the convexity of $K$ it follows that $\rho_{K}(z)>r$ for every $z$ such that $d\left(z^{\prime}, z\right)<\arccos \left(r / s^{\prime}\right)$. Therefore

$$
M_{r} \supset\left(M_{s^{\prime}}\right) \arccos \left(r / s^{\prime}\right), \forall s^{\prime} \geq r .
$$

Define $r(A)$ by

$$
\mathcal{H}_{n-1}(A)=\sigma_{n-1}(r(A)) .
$$

The Brunn-Minkowski inequality on the sphere (see for instance [BZ], Theorem 9.1.1) asserts that

$$
r\left((A)_{\varepsilon}\right) \underset{10}{\geq} r(A)+\varepsilon
$$


Assume that $m\left(s_{1}\right)=l\left(s_{2}\right)$. Then

$$
\begin{aligned}
\frac{n \kappa_{n}}{2} & \geq m(r) \\
& \geq \mathcal{H}_{n-1}\left(\left(M_{s_{1}}\right) \arccos \frac{r}{s_{1}}\right) \\
& \geq \mathcal{H}_{n-1}\left(\left(L_{s_{2}}\right) \arccos \frac{r}{s_{1}}\right) .
\end{aligned}
$$

On the other hand $n \kappa_{n} / 2=l(r)=\mathcal{H}^{n-1}\left(\left(L_{s_{2}}\right) \arccos \frac{r}{s_{2}}\right)$ and thus $\arccos \frac{r}{s_{1}} \leq$ $\arccos \frac{r}{s_{2}}$, that is $s_{1} \leq s_{2}$.

Therefore $m(s) \leq l(s)$ everywhere and then $\operatorname{vol}(K) \leq \operatorname{vol}(C)$. Hence, in order to conclude the proof, it is enough to show that $\operatorname{vol}(C) \leq \operatorname{vol}\left(((R+r) / 2) B_{n}\right)$. By Fubini's Theorem, we can write

$$
\begin{aligned}
\operatorname{vol}(C) & =\int_{r B_{n} \cap w^{\perp}}\left(\sqrt{R^{2}-|y|^{2}}+\sqrt{r^{2}-|y|^{2}}\right) d y \\
& =(n-1) \kappa_{n-1} \int_{0}^{r} s^{n-2}\left(\sqrt{R^{2}-s^{2}}+\sqrt{r^{2}-s^{2}}\right) d s \\
& \leq(n-1) \kappa_{n-1} \int_{0}^{r} s^{n-2} 2 \sqrt{\left(\frac{R+r}{2}\right)^{2}-s^{2} d s} \\
& \leq(n-1) \kappa_{n-1} \int_{0}^{\frac{R+r}{2}} s^{n-2} 2 \sqrt{\left(\frac{R+r}{2}\right)^{2}-s^{2}} d s \\
& =\operatorname{vol}\left(\frac{R+r}{2} B_{n}\right),
\end{aligned}
$$

where we have used the concavity of $\sqrt{x^{2}-s^{2}}$ as a function of $x$.

Let $T$ be a $\delta$-net for some $\delta>0$ and let $t=|T|$. Let $\alpha \in(0,1), \delta>0, t$, $f \in[0, t] \cap \mathbb{N}$ and $a>0$ be so that

$$
1-t^{m}\left(1-\alpha^{2^{m}}\right)^{\left[t /(2 b(n)+2)^{m}\right]}-\sum_{i=f}^{t}\left(\begin{array}{l}
t \\
i
\end{array}\right) \alpha^{i}(1-\alpha)^{t-i}>0
$$

and

$$
f \sigma_{n-1}(6 \delta m+a) \leq \frac{n \kappa_{n}}{2}
$$

As will be clear in the proof of Theorem 3, we are interested in choosing the parameters which (asymptotically in $m$ ) maximize $a$ under the constraints (5.3) and (5.4).

Lemma 10. Let $n$ be fixed. For any $\beta>0$ it is possible to choose parameters $\alpha, \delta, f$ and $a$ that satisfy (5.3) and (5.4) and with

$$
a \geq \frac{1}{m^{2^{m}(1+\beta)}}
$$

as $m \rightarrow \infty$. 
Proof. Let $f=\alpha t$. In order to maximize $a$ it is not convenient to let $\alpha \rightarrow 1$; thus in what follows we suppose $\alpha<1 / 2$. Let $\phi$ denote $1-\sum_{i=\alpha t}^{t}\left(\begin{array}{l}t \\ i\end{array}\right) \alpha^{i}(1-\alpha)^{t-i}$. Then (5.3) is equivalent to

$$
\frac{t}{\log t}>\frac{m(2 b(n)+2)^{m}}{-\log \left(1-\alpha^{2^{m}}\right)}\left(1-\frac{\log \phi}{m \log t}\right) .
$$

This formula shows that in order to satisfy (5.3), not only $t$ but also (multiply both sides by $\alpha$ ) $\alpha t$ has to tend to infinity as $m \rightarrow \infty$. Therefore $\phi$, the probability that an $\alpha$-Bernoulli random variable is less than or equal to its average, tends to $1 / 2$. We can thus conclude that defining a function $\tilde{t}(\alpha, n, m)$ by

$$
\tilde{t}(\alpha, n, m)=\left(\frac{2 m(2 b(n)+2)^{m}}{-\log \left(1-\alpha^{2^{m}}\right)}\right)^{1+\beta / 2},
$$

inequality (5.3), with $f=\alpha t$, is satisfied for any $t>\tilde{t}(\alpha, n, m)$.

We recall that, for any $\delta, t$ is bounded from above and from below respectively by the functions $\bar{t}(\delta)$ and $\underline{t}(\delta)$ defined in (3.3). In the rest of the proof let $\delta=\delta(\alpha, n, m)$ be defined implicitly by the relation $\underline{t}(\delta)=\tilde{t}(\alpha, n, m)$. With this choice of $\delta,(5.3)$ is satisfied.

Since $f=\alpha t \rightarrow \infty$ as $m \rightarrow \infty,(5.4)$ holds only if $6 m \delta+a \rightarrow 0$. For small values of $6 m \delta+a$ it is (see $(3.2)$ )

$$
\sigma_{n-1}(6 m \delta+a) \leq 2 \sin ^{n-1}(6 m \delta+a) \kappa_{n-1} .
$$

Therefore choosing

$$
a=\arcsin \left(\left(\frac{c(n)}{4 \alpha \bar{t}(\delta)}\right)^{\frac{1}{n-1}}\right)-6 m \delta,
$$

both (5.3) and (5.4) are satisfied. We recall that $c(n)=n \kappa_{n} / \kappa_{n-1}$. The only parameter that is left to choose is $\alpha$; we choose $\alpha=\alpha(m, n)=1 / 4\left(\frac{2^{m}-1}{6 m 2^{m}}\right)^{n-1}$. This choice maximizes (5.6) asymptotically in $m$.

Let us now study the asymptotic behaviour of $a$ as $m \rightarrow \infty$. It is convenient to express the dependence of $\delta(\alpha)$ and $\bar{t}(\delta(\alpha))$ on $\alpha$. We have

$$
\delta(\alpha)=\frac{1}{2} \arcsin \left(\left(\frac{c(n) \cos (2 \delta)}{\tilde{t}(\alpha)}\right)^{\frac{1}{n-1}}\right)
$$

and

$$
\bar{t}(\delta(\alpha))=\frac{\tilde{t}(\alpha) 2^{n-1}}{\cos (2 \delta)}\left(1-o_{1}(\alpha)\right)
$$

where $o_{1}(\alpha)=\frac{n-1}{8}\left(\frac{c(n) \cos (2 \delta)}{\tilde{t}(\alpha)}\right)^{\frac{2}{n-1}}(1+o(1))$. Thus as $m \rightarrow \infty$ we have

$$
\begin{aligned}
a & =\arcsin \left(3 m \frac{2^{m}}{2^{m}-1}\left(\frac{c(n) \cos (2 \delta)}{\tilde{t}(\alpha)\left(1+o_{1}(\alpha)\right)}\right)^{\frac{1}{n-1}}\right) \\
& -3 m \arcsin \left(\left(\frac{c(n) \cos (2 \delta)}{\tilde{t}(\alpha)}\right)^{\frac{1}{n-1}}\right) \\
= & \frac{3 m}{2^{m}-1}\left(\frac{c(n)}{\tilde{t}(\alpha)}\right)^{\frac{1}{n-1}}(1+o(1)) .
\end{aligned}
$$


We now substitute the expression of $\tilde{t}(\alpha)$ that comes from (5.5), the expression of $b(n)$ that comes from $(3.4)$ and the known estimate $c(n)=\sqrt{2 \pi n}(1+o(1))$. We obtain

$$
a \geq 3 m\left(\left(\frac{\sqrt{2 \pi n}}{m 2^{m+1} 4^{2^{m}}}\right)^{\frac{1}{n-1}} \frac{1}{e 6^{m}} \frac{1}{(6 m)^{2^{m}}}\right)^{1+\beta / 2}(1+o(1)) .
$$

Clearly this implies the statement of the lemma.

Proof of Theorem 3. Let $T$ be a $\delta$-net and $t=|T|$. Let $\alpha \in(0,1), \delta>0, t$, $f \in[0, t] \cap \mathbb{N}$ and $a>0$ be parameters that satisfy (5.3) and (5.4). By Lemma 6 and Lemma 7 , there exists a subset $A^{\prime}$ of $T$ such that $\left(A^{\prime}\right)_{6 m \delta}$ satisfies the $m$-orbit property. Let $H_{m}$ be defined as the convex hull of $r S^{n-1}$ and of $R\left(A^{\prime}\right)_{6 m \delta}$, with $r>0$ and $R>0$ chosen so that $r / R=\cos a$ and moreover $\operatorname{vol}\left(H_{m}\right)=\kappa_{n}$. By construction the outer radius of $H_{m}$ is not decreased by any $m$ symmetrizations.

We claim that $\mathcal{H}_{n-1}\left(H_{m} \cap s S^{n-1}\right) \leq n \kappa_{n} s^{n-1} / 2$ for every $s \in(r, R]$. By the relation $r / R=\cos a$ and (5.2) it follows easily that every point of $r S^{n-1} \backslash r\left(A^{\prime}\right)_{6 m \delta+a}$ is contained in the boundary of $H_{m}$. Hence by (5.4) we deduce

$$
\begin{aligned}
\frac{\mathcal{H}_{n-1}\left(H_{m} \cap s S^{n-1}\right)}{s^{n-1}} & \leq \frac{\mathcal{H}_{n-1}\left(\stackrel{\circ}{H}_{m} \cap r S^{n-1}\right)}{r^{n-1}} \\
& \leq \mathcal{H}_{n-1}\left(\left(A^{\prime}\right)_{6 m \delta+a}\right) \leq\left|A^{\prime}\right| \sigma_{n-1}(6 m \delta+a) \\
& \leq f \sigma_{n-1}(6 m \delta+a) \leq n \kappa_{n} / 2, \forall s \in(r, R]
\end{aligned}
$$

where $\stackrel{\circ}{H}_{m}$ denotes the interior of $H_{m}$.

We can now apply Lemma 9 to conclude that the Hausdorff distance from $H_{m}$ to $B_{n}$ is attained by the outer sphere.

This distance is

$$
d_{H}\left(H_{m}, B_{n}\right) \geq \frac{R-r}{2}=\frac{R}{2}\left(1-\frac{r}{R}\right)=\frac{R}{2}(1-\cos a) \geq \frac{a^{2}}{6}
$$

as $m \rightarrow \infty$. One can use Lemma 10 to estimate $a$; certainly

$$
a \geq \frac{1}{m^{2^{m}(1+\beta)}} .
$$

Thus

$$
d_{H}\left(H_{m}, B_{n}\right) \geq \frac{1}{6 m^{22^{m}(1+\beta)}} .
$$

Let us denote $d_{H}\left(H_{m}, B_{n}\right)$ by $\varepsilon(n, m)$. By definition of $N(n, \varepsilon)$, it is certainly true that $N(n, \varepsilon(n, m)) \geq m$. Expressing $m$ in terms of $\varepsilon(n, m)$ one gets that as $m \rightarrow \infty$

$$
N(n, \varepsilon(n, m)) \geq \frac{\log \left(\log \left(\frac{1}{\varepsilon(n, m)}\right)\right)}{\log (2)}(1+o(1)) .
$$

Since $N$ is monotone, this concludes the proof.

Acknowledgments. The authors would like to thank Imre Bárány for pointing them to the probabilistic result by Erdös quoted in the paper and Joram Lindenstrauss for many useful comments on the first version of this paper. 


\section{REFERENCES}

[BLM] J. Bourgain, J. Lindenstrauss and V. Milman, Estimates related to Steiner symmetrizations, GAFA 87-88 (J. Lindenstrauss, V. Milman, eds.), Lecture Notes in Math. 1376, Springer, Berlin, 1989, pp. 264-273.

[BZ] Yu. D. Burago, V. A. Zalgaller, Geometric inequalities, Springer, Berlin, 1988.

[FTK] G. Fejes Tòth, W. Kuperberg, Packing and coverings with convex sets, Handbook of Convex Geometry (P. M. Gruber, J. M. Wills, eds.), North-Holland, Amsterdam, 1993, pp. 799-860.

[H1] H.Hadwiger, Einfache Herleitung der isoperimetrischen Ungleichung für abgeschlossen Punktmengen, Math. Ann. 124 (1952), 158-160.

[H2] H.Hadwiger, Vorlesungen über Inhalt, Oberfläche und Isoperimetrie, Springer, Berlin, 1957.

[HR] H. Halberstam, K. F. Roth, Sequences, Oxford University Press, London, 1966.

[T] A. Tsolomitis, Quantitative Steiner/Schwarz-type symmetrizations, Geometriae Dedicata 60 (1996), 187-206.

G. Bianchi, Dipartimento di Matematica, Università di Ferrara, Via Machiavelli 35, I-44100 Ferrara, ITALY

E-mail address: bia@unife.it

P. Gronchi, Istituto di Analisi Globale ed Applicazioni, CNR Via S. Marta 13/A, I-50139 FiRENZE, ITALY

E-mail address: paolo@iaga.fi.cnr.it 